\title{
Evolving our support for early sharing
}

\author{
Nature Communications encouraged rapid dissemination of results with the launch of Under Con- \\ sideration in 2017. Today we take one more step by offering an integrated preprint deposition service
} to our authors as part of the submission process.

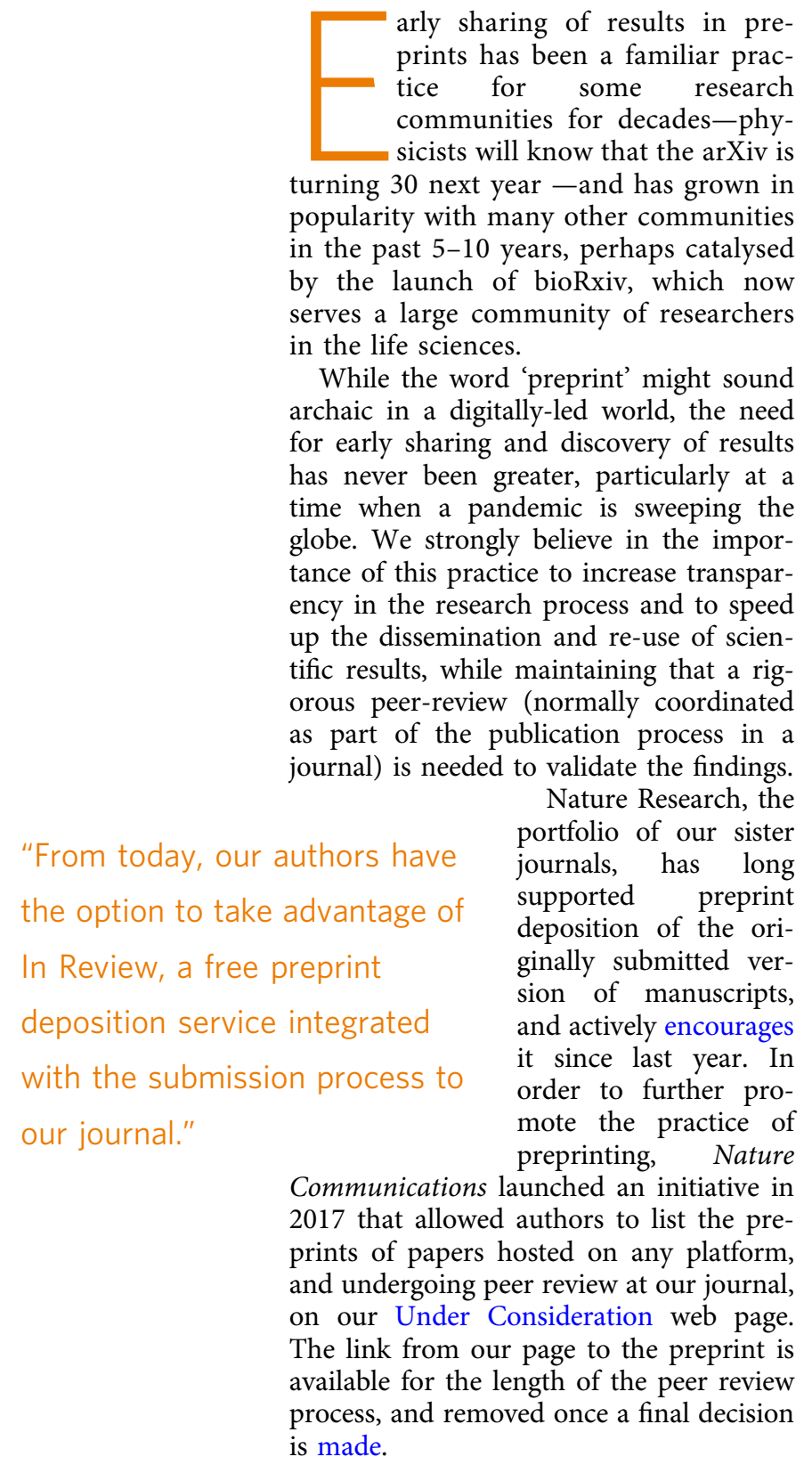

We have been offering this option to our authors for two and a half years, and we are very happy to report that $59 \%$ of the authors who opted in ended up depositing a preprint of their work as a result of our initiative. The overall uptake has grown from $3 \%$ in 2017 to $7 \%$ in 2019 , and has varied significantly by discipline, with $22 \%$ of participating papers in the physical sciences, and $78 \%$ in the life and biological sciences. Perhaps this uneven breakdown is a reflection of the more recent adoption of preprints by the latter community, when compared with the more mature practice in the physical sciences. Authors working in neuroscience have been by far the most receptive to this initiative, contributing $18 \%$ of all participating papers.

Wishing to facilitate greater adoption of preprints across the multidisciplinary scope of our journal, we are retiring Under Consideration to support a new early sharing tool with added functionalities for our authors.

From today, our authors have the option to take advantage of In Review, a free preprint deposition service integrated with the submission process to our journal. The preprint of the author's original submission will be posted (with a permanent DOI, under a CC-BY licence) on the multidisciplinary platform hosted by our partner Research Square at the same time as the submission is being considered by our editorial team.

For authors who opt-in, the posting will happen when our editors decide whether to send the manuscript for external review. If the manuscript is sent to reviewers, the preprint on Research Square will be displayed as being 'under review at Nature Research' for as long as the manuscript is being considered. Once the manuscript is published, the preprint will link to the published version. When the preprint is 
posted, a HTML version of the manuscript will be created and displayed when original figures are available, making it more machine-readable - and hence easier to be discovered by search engines-than a PDF.

As part of the service offered by In Review, authors will be able to track the status of their manuscript thanks to a private peer review timeline, showing main events such as the recruitment of a reviewer or the receipt of a reviewer report. More information about In Review for Nature Research journals, including the minimum disclosures required, can be found here. At present, the In Review opt-in is available for submissions received via the Nature Communications portal, but not to authors transferring their paper from another Nature Research journal.

In Review has been offered on almost 300 Springer Nature journals since October
2018. This integrated preprint deposition service will hopefully make it easier, and thus more likely, for our authors to share their results early. Moreover, we believe that In Review will be particularly useful in serving communities that do not yet have an established discipline-specific preprint server, as well as providing more visibility for multidisciplinary works.

We continue to encourage preprint deposition on any community-recognised preprint server-it is solely the author's choice to decide if and where they are going to post their results before the conclusion of the peer review process.

We are excited about this new step we are taking in support of early sharing. We will monitor reception of this new service by our authors, and continue to evolve our offering to them in response to the needs of the community.
Published online: 15 June 2020

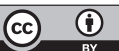

Open Access This article is licensed under a Creative Commons Attribution 4.0 International License, which permits use, sharing, adaptation, distribution and reproduction in any medium or format, as long as you give appropriate credit to the original author(s) and the source, provide a link to the Creative Commons license, and indicate if changes were made. The images or other third party material in this article are included in the article's Creative Commons license, unless indicated otherwise in a credit line to the material. If material is not included in the article's Creative Commons license and your intended use is not permitted by statutory regulation or exceeds the permitted use, you will need to obtain permission directly from the copyright holder. To view a copy of this license, visit http:// creativecommons.org/licenses/by/4.0/.

(C) Springer Nature Limited 2020 